\title{
Single-cell discrimination based on optical tweezers Raman spectroscopy
}

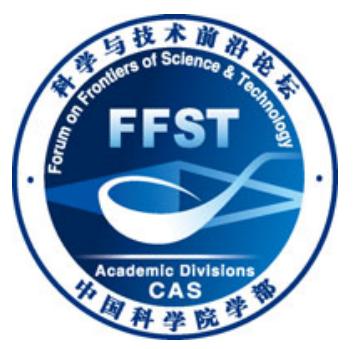

\author{
MA HongFei ${ }^{1 \dagger}, \mathrm{ZHANG}^{\mathrm{Yong}^{2 \dagger}} \& \mathrm{YE} \mathrm{AnPei}^{1^{*}}$ \\ ${ }^{1}$ Key Laboratory for the Physics and Chemistry of Nanodevices, School of Electronics Engineering and Computer Science, \\ Peking University, Beijing 100871, China; \\ ${ }^{2}$ Beijing Institute of Biomedicine, Beijing 100091, China
}

Received September 21, 2012; accepted November 8, 2012; published online March 12, 2013

The ability to discriminate between single cells in a label-free and noninvasive fashion is important for the classification of cells, and for the identification of similar cells from different origins. In this paper, we present the Raman spectroscopy-based identification of different types of single cells in aqueous media, and discrimination between the same types of cells from different donors using a novel Laser Tweezers Raman Spectroscopy (LTRS) technique, which combines laser trapping and micro-Raman spectroscopy. First, we measured the spectra of individual living human erythrocytes, i.e. red blood cells, and leucocytes (U937 cancer cells). High-quality Raman spectra with low fluorescence were obtained using a home-LTRS apparatus and 20 cells were measured for each cell type. The smoothing, baseline subtraction, and normalization of the data were followed by a principal components analysis (PCA). The PCA loading plots showed that the two different types of cells could be completely separated based only on the first component (PC1) (i.e. the peaks at $1300 \mathrm{~cm}^{-1}$ ); the discrimination accuracy could therefore reach $100 \%$. More than 50 spectra were taken for each erythrocyte obtained from the four healthy volunteers. The average discrimination accuracy was $84.5 \%$ for two random individuals taken from the four volunteers, according to the first and second PCs. This work demonstrates that LTRS is a powerful tool for the accurate identification and discrimination of single cells, and it has the potential to be applied for the highly sensitive identification of cells in clinical diagnosis and medical jurisprudence.

Raman spectroscopy, optical tweezers, human erythrocyte, single cell identification, principle components analysis

Citation: $\quad$ Ma H F, Zhang Y, Ye A P. Single-cell discrimination based on optical tweezers Raman spectroscopy. Chin Sci Bull, 2013, 58: 2594-2600, doi: 10.1007/ s11434-013-5721-6

Raman spectroscopy is a powerful analytical technique that can be used to directly i.e. without exogenous labeling and non-invasively detect molecular composition and distribution in a studied sample, based on the measurement of the specific vibration modes of molecules. Raman spectroscopy is widely used in physics, chemistry, biology, and related interdisciplinary branches of science. Many biochemical processes such as cell division, cell differentiation, cell signaling, and phagocytosis are accompanied by a large-scale spatial reorganization of the molecular components that constitute the cell. Micro-Raman spectroscopy has been

$\dagger$ These authors contributed equally to this work.

*Corresponding author (email: yap@pku.edu.cn) used for single living cell chemical analysis [1-4], and Raman imaging [5-7]. Single cell vibration Raman spectroscopy has been proven to be sufficiently sensitive to allow the measurement of the typical spectra of the cell nucleus [8] and cell cytoplasm, achievements that confirm the feasibility of single-cell identification. However, there is a large class of living cells including red blood cells that must be suspended in an aqueous environment for their normal functionality to be retained. Brownian motion, motility, and fluxes in the surrounding media may cause the living cells to move away from the region of excitation during the relatively long Raman acquisition times, meaning that the single cell may not be fully excited while the spectra are collected. Conventional methods for resolving this problem 
involve the use of a micropipette, or fixing the cell on a cover slip via chemical or physical methods. In many cases, however, this may induce undesirable perturbations, such as reflections from the cover slip. Moreover, chemical immobilization may change the microenvironment of the living cells and may yield unknown effects, especially in studies on the surface of living cells.

An elegant approach for the immobilization of micrometer-sized particles uses the radiation gradient force produced by a strongly focused laser beam i.e. optical tweezers to stably confine the floating cell in its natural environment. An additional advantage of this approach is that it permits one to directly probe the individual cell, and obtain important information that might be lost in an ensembleaveraged measurement. Since both micro-Raman spectrometry and optical tweezers require a laser beam to be tightly focused using a high numerical aperture objective lens, they can be combined easily in a single system; a so-called Laser Tweezers Raman Spectrometer (LTRS). Recently, LTRS technology has been applied to study microorganisms $[9,10]$, blood cells [11-14], and, to a lesser extent, malignant cells [15-17].

Different types of cells exhibit differences in chemical composition and molecular structure, and even the same type of cells derived from different donors show small divergences, due to genetic variations; this difference is accompanied by changes in a variety of biomolecules, which can be probed using Raman spectroscopy. Recently, some single-cell identification studies have reported the discrimination of different types of cells. For example, in combination with statistical methods, Raman microscopy has been successfully applied to distinguish between benign and malignant cells [18-20], and healthy cells and dying or stressed cells [21]; it has also been used to discriminate between cell types [22-25], even for highly similar cancer cell lines. However, most previous studies focused on distinguishing between different types of cells such as cancer cells and healthy cells, or different states of the same cells. The question of how to discriminate between the same types of cells from different donors for example, red blood cells from different, healthy persons in a label-free, non-invasive fashion remains a challenge. Because the changes in the biochemical composition and structure are small, the changes in the Raman spectra are very weak for the same type of cells derived from different donors. In this paper, making use of the LTRS technique and PCA statistical methods, we first measured the spectra of single U937 cells, and human red blood cells. After verifying the feasibility of distinguishing these two different cell types using our homemade LTRS setup and PCA statistical methods, we demonstrated the reliable discrimination of red blood cells donated from different healthy persons, using the LTRS and PCA approach.

\section{Methods and materials}

\subsection{LTRS setup configuration}

Figure 1 illustrates the optical configuration for our homemade backscattering LTRS system. This setup was based on an inverted microscope (Zeiss, Axiovert 200). An $\mathrm{Ar}^{+}$-ion laser (Shanghai AiAo, ILT5500ASL) delivered Raman

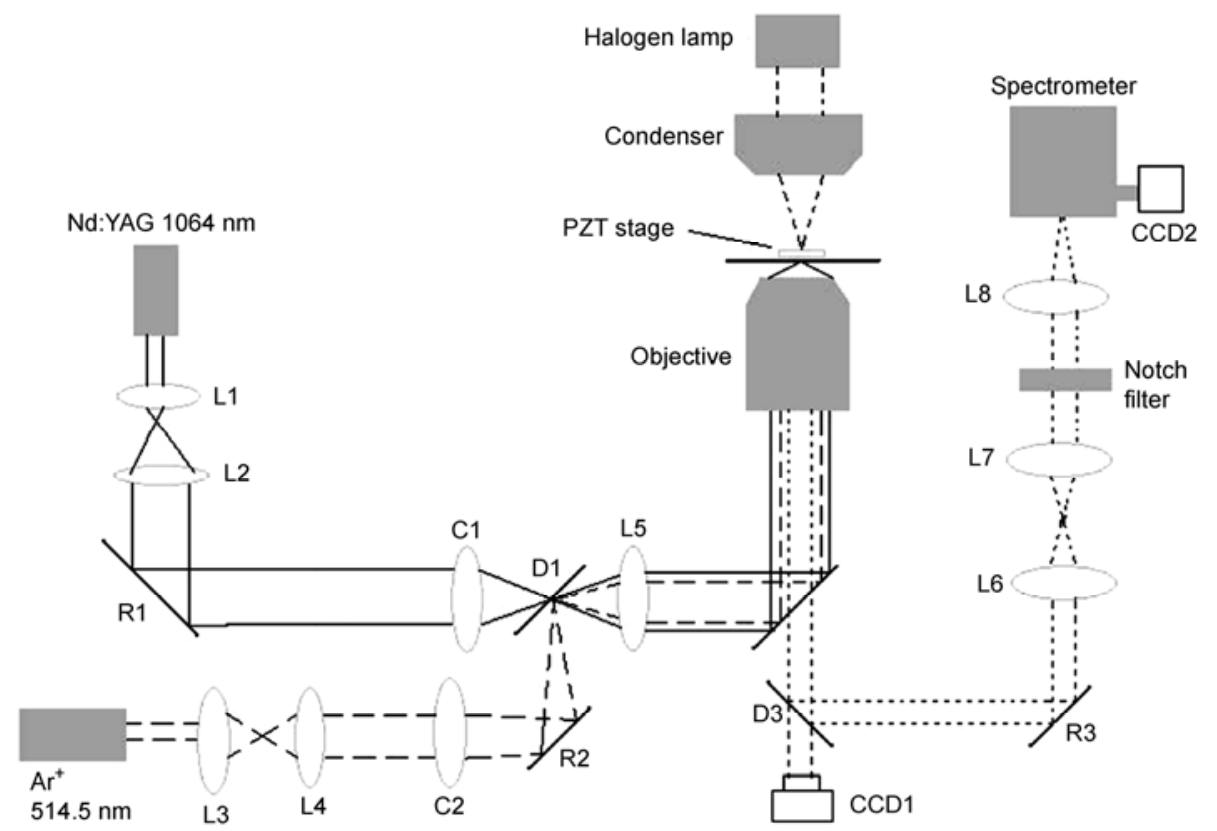

Figure 1 Diagram of the configuration of the LTRS setup. The near-infrared laser $(\lambda=1064 \mathrm{~nm})$ and visible laser $(\lambda=514.5 \mathrm{~nm})$ beams were expand by lenses L1-L4, combined and coupled with the microscope by lenses C1 and C2, and dichroic mirrors D1 and D2, respectively. Raman scattering light was delivered by D3 and mirror R3 into the spectrometer, and the spectra were collected by a cooled spectroscopic CCD. 
excitation at a wavelength of $514.5 \mathrm{~nm}$, with a power of 10 $\mathrm{mW}$ (at the entrance of the objective). The trapping laser used was a $1064 \mathrm{~nm}$ Nd:YAG laser (Coherent, Compass 1064-2000N), with a power of approximately $40 \mathrm{~mW}$ at the objective back aperture. Both lasers passed through two telescope arrangements of lenses (L1-L4), to expand the laser beams. Both the exciting light and the trapping light were directed to the microscope via dichroic mirrors (D1 and D2), and were coupled into the same objective (Zeiss, $100 \times, \mathrm{NA}=1.3$, oil immersion). The back scattering Raman light was collected by the same objective, and then delivered into the spectrometer (Acton, SpectaPro 2300i) via dichroic mirror D3. A notch filter $(514 \mathrm{~nm})$ was placed before the entrance of spectrometer to filter the Rayleighscattered light from the sample, and other optical noise along the optical path. Raman spectra ranging from 400 to $2200 \mathrm{~cm}^{-1}$ were recorded with a spectral resolution of $4 \mathrm{~cm}^{-1}$, using liquid nitrogen (LN)-cooled spectroscopic charge-coupled device (CCD) (PI, Spec-10). The integration time of the CCD was set to 40s for each spectral collection.

\subsection{Cell preparation}

The human monocytic U937 cell line was provided by the Third Hospital of Peking University. The cells were cultured at $1 \times 10^{6}$ cells $/ \mathrm{mL}$ in RPMI 1640 medium supplemented with $100 \mu \mathrm{g} / \mathrm{mL}$ of penicillin/streptomycin and $10 \%$ fetal calf serum, at $37^{\circ} \mathrm{C}$, in a humidified atmosphere $(5 \%$ $\mathrm{CO}_{2}$ in air). The cells were centrifuged ( $1 \mathrm{~min}, 4000 \mathrm{r} / \mathrm{min}$ ) and harvested, and then washed twice in RPMI 1640 medium without phenol red. The red blood cells were provided by Hospital of Peking University. These peripheral blood samples were collected from 4 people (marked as sample 1 to sample 4), and EDTA was immediately added, to act as a thrombin inhibitor. Before experiments, the blood samples were diluted to 100 times in a phosphate buffer (isotonic, $\mathrm{pH}$ 7.4) to avoid cell adhesion during the spectral measurement processes.

\subsection{Data processing}

Following spectral acquisition, each spectrum was processed using a self-developed spectral processing software based on Matlab (Mathworks, Inc.); this software performed Savisky-Golay 9 points smoothing [26], fluorescence subtraction based on a widely used algorithm [27] and normalization on the spectral data. Principal components analysis (PCA) was then applied. Finally, the deviation from the total sample group was computed for each cell. The results are listed in Table 1.

Generally, in spectroscopic analysis there are so many peaks that it makes it difficult to concisely describe data features using only one or two peaks. Moreover, these less important peaks sometimes disturb the analysis. Briefly, PCA is a statistical method of data processing that reduces the number of variables. It extracts the important peaks and abandons less important peaks through a linear transformation, and offers a set of fewer variables that contains information equivalent to that in the original data $[28,29]$. For instance, we could consider a spectrum with $p$ characteristic peaks as a $p$-dimensional random vector $X=\left(x_{1}, x_{2}, \cdots x_{p}\right)$, where $x_{i}$ is the ith peak's intensity. To execute PCA, we first transform the random vector $X$ to another random vector $Z$, as follows:

$$
\left\{\begin{array}{c}
z_{1}=a_{11} x_{1}+a_{21} x_{2}+\cdots a_{p 1} x_{p}, \\
z_{2}=a_{12} x_{1}+a_{22} x_{2}+\cdots a_{p 2} x_{p}, \\
\vdots \\
z_{p}=a_{1 p} x_{1}+a_{2 p} x_{2}+\cdots a_{p p} x_{p} .
\end{array}\right.
$$

If these coefficients $a_{i j}$ are chosen such that the variance $\sigma_{i}^{2}$ of $z_{i}$ is as large as possible- that is, the quantity of information included in these $z_{i}$ is at a maximum-we can pick $n(n<p)$ such that $z_{i}$ represents original $p$-characteristic-peaks-spectra with $n$ new "peaks". It has been shown that these coefficients $a_{i j}$ can be obtained by solving the covariance matrix $\Sigma$ of the random vector $X$ [28].

$$
\sum=\left(\begin{array}{ccc}
\operatorname{Cov}\left(x_{1}, x_{1}\right) & \cdots & \operatorname{Cov}\left(x_{1}, x_{p}\right) \\
\vdots & \ddots & \vdots \\
\operatorname{Cov}\left(x_{p}, x_{1}\right) & \cdots & \operatorname{Cov}\left(x_{p}, x_{p}\right)
\end{array}\right)
$$

where, $\operatorname{Cov}\left(x_{i}, x_{j}\right)=E\left[\left(x_{i}-E\left(x_{i}\right)\right)\left(x_{j}-E\left(x_{j}\right)\right)^{\prime}\right]$ is the covariance, and $E$ represents the expectation value. By resolving the eigen equation, the eigenvalues $\lambda$ and eigenvectors $I$ can be acquired. The $\lambda_{i}$ correspond to $\sigma_{i}^{2}$, while the $I_{i}$ correspond to a group of coefficients $\left(a_{i 1}, a_{i 2}, \cdots a_{i p}\right)$. If we sequence these $\lambda_{i}$ as $\lambda_{1}>\lambda_{2}>\cdots>\lambda_{p}$, the first $z_{1}$ (corresponding to the biggest $\lambda_{1}$ ) is called Principal Component 1 (PC1), which offers the greatest amount of information. In addition, it is possible to check how much information is included in these PCs. The quantity of information for the $i$ th PC can be measured using the variance explained, $\eta_{i}$ which is defined as [28]

Table 1 Identification accuracy rates

\begin{tabular}{cccccc}
\hline Sample 1 vs. sample 2 & Sample 1 vs. sample 3 & Sample 1 vs. sample 4 & Sample 2 vs. sample 3 & Sample 2 vs. sample 4 & Sample 3 vs. sample 4 \\
\hline $76.6 \%$ & $87.3 \%$ & $98.2 \%$ & $84.5 \%$ & $86.4 \%$ & $74.1 \%$ \\
\hline
\end{tabular}




$$
\eta_{i}=\frac{\sigma_{i}^{2}}{\sum_{j} \sigma_{j}^{2}} .
$$

The total variance explained is defined as $\sum_{i=1}^{n} \eta_{i}$, which tells us the total quantity of information corresponding to the totality of the selected PCs. A detailed discussion on this subject can be found in ref. [28].

\section{Results and discussion}

\subsection{Comparison between U937 and erythrocyte groups}

Figure 2(a) shows typical Raman spectra profiles for U937 and erythrocyte cells (red blood cells). The difference between the U937 and red blood cells was observable, even without the assistance of mathematic processing, and this difference was considerably larger than that observed for interclass cells. To verify the reliability of this statistical method, we randomly selected spectra for 20 U937 cells, and 20 erythrocyte cells. Some of the main peaks used in
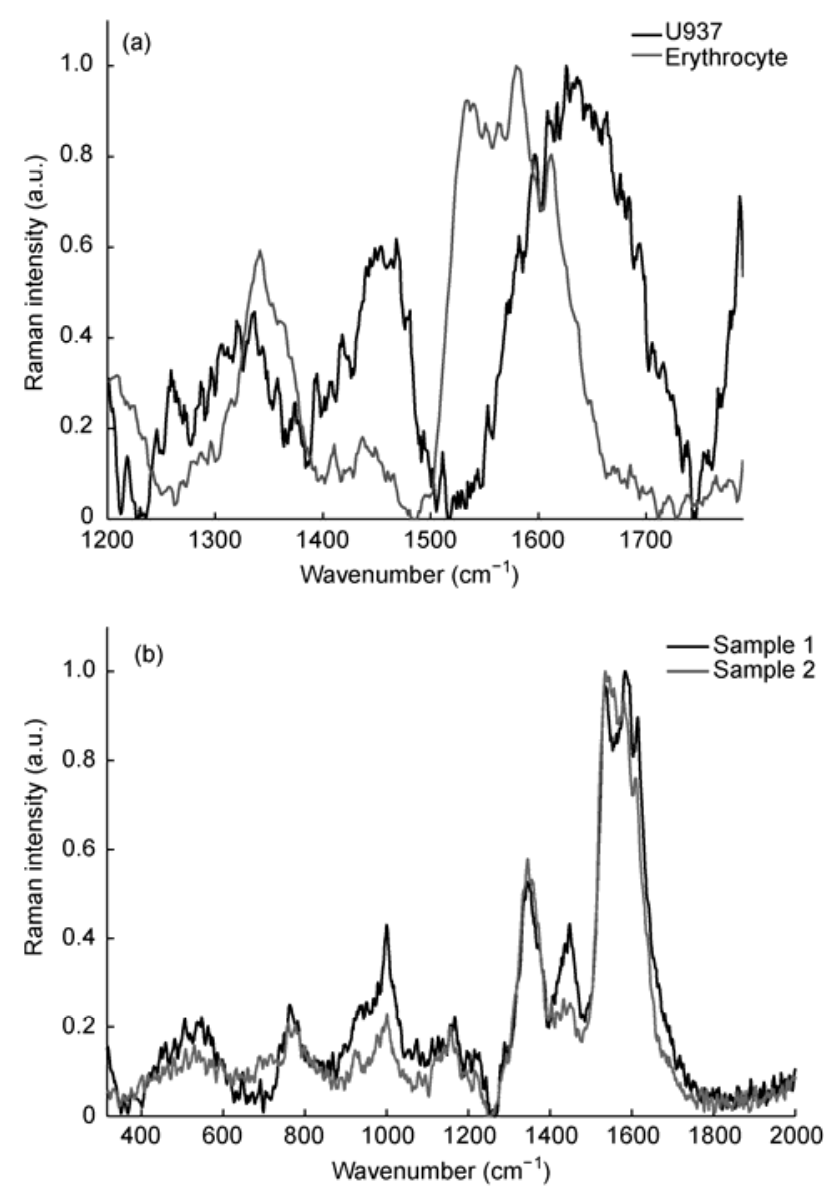

Figure 2 Normalized spectra for single cells. (a) Spectra for U937 and erythrocytes. (b) Erythrocyte spectra from different donors. the comparison are listed in Table 1 . They were assigned by referring to previous reliable studies [30,31]. For U937, the large peaks at 1445 and $1654 \mathrm{~cm}^{-1}$ were selected, while for the erythrocytes, the main characteristic peaks for hemoglobin at 1342 and $1526 \mathrm{~cm}^{-1}$ were chosen.

As shown in Figure 2(a), the two spectra were distinguishable by their peaks at $1342,1445,1526 \mathrm{~cm}^{-1}$ and so on, but it was impractical to analyze so many peaks manually. Here, we adopted PCA to extract the primary differences. The results are illustrated in Figure 3. Five peaks were used to perform PCA; these were at 1300, 1342, 1445, 1600, and $1654 \mathrm{~cm}^{-1}$. Each point in the PCA plot represents one spectrum or one cell; 40 cells were compared. PCA transformed the five peaks into two-dimensional coordinates when the 1 st and 2nd principal components (PC) were taken into account. In most cases, it could be assumed that the first two PC components would concentrate almost all of the information contained by the five peaks. For this five-peak PCA, the total variance explained of the 1st and 2nd PCs was $94.8 \%$. This meant that the 1 st and 2nd PCs included $94.8 \%$ of the information contained in the original five peaks. The PCA plot showed that the two types of cell were completely separated by the 1 st PC. In investigating the spectral data, we found that the $1 \mathrm{st} \mathrm{PC}$ was mainly composed of the peaks at 1342 and $1300 \mathrm{~cm}^{-1}$. That is to say, the two cell types could be separated using these peaks. This result is reasonable, because the peak at $1342 \mathrm{~cm}^{-1}$ was ascribed to porphyrin vibrations, and this vibration mode is unique to red blood cells. Although a nucleic acid band was also located at this wavelength, the U937 nucleic acid peak was weaker than the red blood cell porphyrin peak, due to its resonance Raman scattering [31,32]. The 2nd PC mainly consisted of the $1445 \mathrm{~cm}^{-1}$ peak, which could be assigned as representing the amount of lipids. The peak dispersion of U937 along the 2nd PC was larger than that of the red blood cells. This suggested that the lipid content in the red blood cells was higher than that in U937, perhaps because the U937 cells

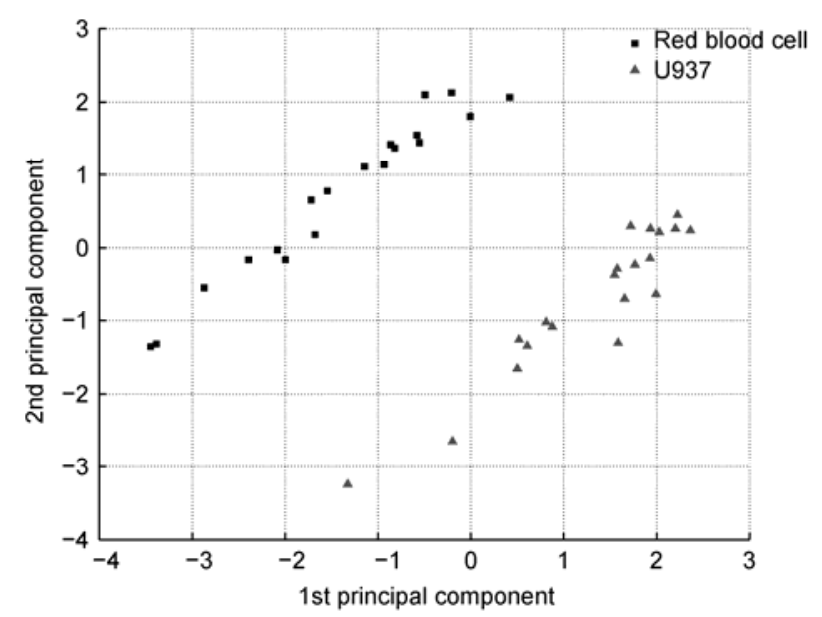

Figure 3 PCA plot for U937 and red blood cells. Each point in the PCA plot represents one spectrum or cell. 
might have been in a different cell cycle, while a mature red blood cell would never divide.

\subsection{Interclass identification of erythrocytes}

Figure 2(b) shows two normalized spectra for red blood cells originating from samples 1 and 2 . Although they were the same kind of cell, their spectral profiles were different; the peaks at 1000,1442 , and $1550 \mathrm{~cm}^{-1}$ were remarkably diverse. To set up the criteria for the identification of a cell's origin, it was necessary to compare all of the useful peaks from two arbitrary sample populations, and reveal the differences. Figure 4 shows scatter plots for the 1st PC score versus the 2 nd PC score, with each plot containing data for 110 cells (half and half for each group of samples). In consideration of future practical applications, we used the data for these 110 cells to create a database. New cells can now be compared with the database, and added to it. The following analysis focused on the database itself. The total variance of the two groups of samples exceeded $90 \%$, according to the 1 st and 2nd PCs. As previous mentioned, samples 1 to 4 represented people 1 to 4 . By simply drawing a discrimination line in the PC space coordinates, as in Figure 4(b), the cell type could be assigned. If the point was located above the line, it was classified into sample 1, and if the point was located below the line, the cell was assigned to sample 4. Comparing Figure 4 (a) and (b), it is noticeable that the more peaks were included in PCA plot, the more obvious the separation effect was. In Figure 4 (a), four peaks (at 1000, 1342, 1445, and $1550 \mathrm{~cm}^{-1}$ ) were included in the PCA plot, and $95.4 \%$ of the cells could be accurately identified. As shown in Figure 4(b), three additional peaks (at 1300, 1600, and $1654 \mathrm{~cm}^{-1}$ ) were included in the PCA, so that only two cells from sample 1 were improperly classified as sample 4 , and the identification accuracy reached 98.2\%. This phenomenon is not a special case for twosample identification. However, the mathematical principles of PCA do not assure this conclusion. The precondition is that the peaks we choose should represent actual vibration bands, so that they are actually different from each other. In case of seven-peak PCA, the 1st PC was mainly composed of 1445 and $1654 \mathrm{~cm}^{-1}$ peaks, and the peaks at 1342 and $1550 \mathrm{~cm}^{-1}$ were most important for the 2 nd PC. From Table 2 , we know that the peak at $1445 \mathrm{~cm}^{-1}$ indicated the amount of lipids, while the peak at $1342 \mathrm{~cm}^{-1}$ was characteristic of hemoglobin. Figure 4(b) indicated that samples 1 and 4 were distinguished mainly by their 2nd PCs, while their 1st PCs were similar. Since the spectra were normalized, it was not appropriate to compare the absolute contents of cells from the two sample groups, but we could deduce that the relative protein content (hemoglobin versus lipid) of sample 4 was higher than that of sample 1. A similar trend was also observed in other cases (Figure 4(b) and (c)), for example. In summary, we believe that these differences in the Raman signature derived mainly from differences in the protein-lipid ratio. It is interesting to question what caused
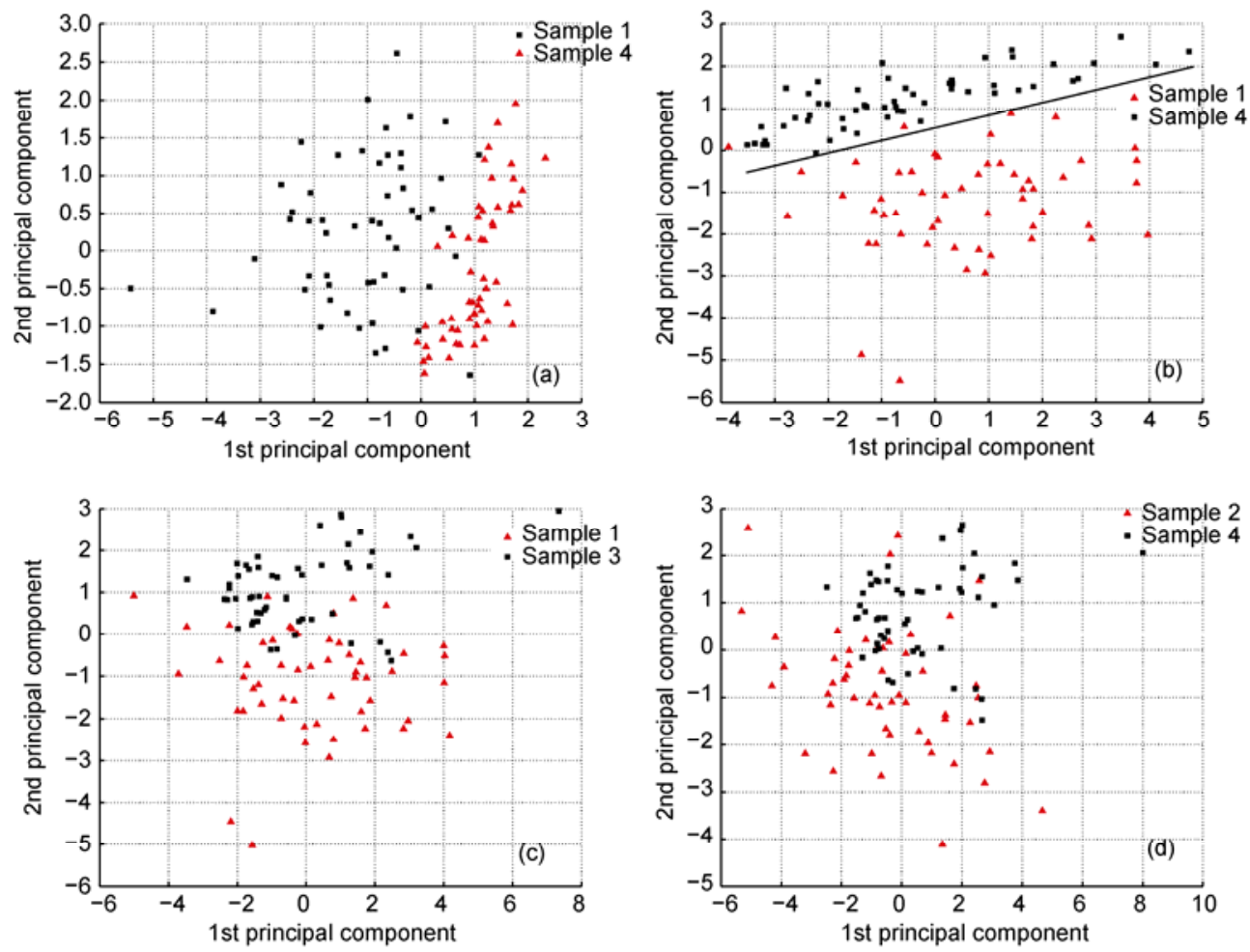

Figure 4 (Color online) PCA plots for different red blood cell samples. (a) Four-peak PCA plot for samples 1 and 4 . (b) Seven-peak PCA plot for samples 1 and 4, with a discrimination line. (c) Seven-peak PCA plot for samples 1 and 3. (d) Seven-peak plot for samples 2 and 4. 
Table 2 Raman shift observed in the spectra of U937 and red blood cells, with tentative assignment

\begin{tabular}{ccc}
\hline \multirow{2}{*}{ Peaks $\left(\mathrm{cm}^{-1}\right)$} & \multicolumn{2}{c}{ Assignment } \\
\cline { 2 - 3 } & U937 & Red blood cell \\
\hline 1000 & Phenylalanine & Phenylalanine \\
1300 & Amide III & Amide III \\
1342 & Nucleic acids $(\mathrm{A}, \mathrm{G})$ & $v(\text { Porphyrin half-ring })_{\mathrm{sym}}$ \\
1445 & $\delta_{\mathrm{CH}_{2} / \mathrm{CH}_{3}}$ & $\delta_{\mathrm{CH}_{2} / \mathrm{CH}_{3}}$ \\
1526 & - & $v_{\mathrm{porphyrin} \text { breathing }}$ \\
1550 & - & $v_{\mathrm{C \beta C \beta}}$ \\
1600 & $v_{\mathrm{C}_{\mathrm{a}}=\mathrm{C}_{\mathrm{b}}}$ & $v_{\mathrm{C}_{\mathrm{a}}=\mathrm{C}_{\mathrm{b}}}$ \\
1654 & Amide I $v_{\mathrm{C}=\mathrm{O}}$ & Amide I $v_{\mathrm{C}=\mathrm{O}}$ \\
\hline
\end{tabular}

the differences in the Raman spectra of red blood cells from different people. We propose two possible reasons for this phenomenon: The first is that the protein-lipid ratios of red blood cells are inherently different for individuals, due to genetic variations. The second is that individual physiological differences such as age, gender, and health condition may affect the protein-lipid ratio. Here, these differences could be used to identify the blood cells. Using seven-peak PCA, we accurately identified all of the samples. The results are listed in Table 1 . The average accuracy rate was $84.5 \%$, which demonstrates that red blood cells from different donors do have inherent differences in their biochemical components.

It is worth noting that in the seven-peak case, the cells derived from different samples were separated by the 2 nd PC. This indicated that the differences between red blood cells were smaller than the differences between different types of cells. To identify the differences between samples, we must correctly select the meaningful peaks that give rise to inter-sample differences; otherwise, the differences will be covered by the full-spectra deviation.

\section{Conclusions}

As a non-destructive, information-rich spectroscopic technique, LTRS has the potential to supplement existing cell identification techniques. It holds advantages in its ability to accurately detect single, floating, living cells in a non-invasive and label-free fashion. Using LTRS, we can not only non-destructively detect and identify the type of single cells, but also separate specific cells from a mixed sample in an aqueous medium, and perform subsequent studies on these cells; stem cell identification and separation is an example of a potential application for this. Based on a home-built LTRS apparatus combined with PCA statistical methods, we studied the spectra of two different types of single cells, imonocytic U937 cells, and human erythrocytes. Preliminary studies verified that the identification accuracy for U937 and erythrocyte cells could reach $100 \%$, while for same type of cells-such as red blood cells derived from different people-the average identification accuracy reached approximately $85 \%$, using the first two PCA components. Although the identification accuracy was not perfect, this method based on biochemical components suggests itself as an alternative to traditional DNA analysis. Compared with DNA sequencing, the main advantages of LTRS identification are its label-free, nondestructive, quick and inexpensive nature. It offers high sensitivity, and has great potential as an approach for single-cell identification.

This work was partly supported by Beijing Natural Science Foundation (5102019) and National Science and Technology Infrastructure Program (2012BAF14B14).

1 Chan J W, Motton D, Rutedge J C, et al. Raman spectroscopic analysis of biochemical changes in individual triglyceride-rich lipoprotein in the pre- and postprandial state. Anal Chem, 2005, 77: 5870-5876

2 Xie C G, Li Y Q. Confocal micro-Raman spectroscopy of single biological cells using optical trapping and shifted excitation difference techniques. J Appl Phys, 2003, 93: 2982-2986

3 Calander N, Willander M. Optical trapping of single fluorescent molecules at the detection spots of nanoprobes. Phys Rev Lett, 2002, 89: 143603

4 Xie C G, De C, Li Y Q. Raman sorting and identification of single living micro-organisms with optical tweezers. Opt Lett, 2005, 30: 1800-1802

5 Creely C, Volpe G, Singh G, et al. Raman imaging of floating cells. Opt Express, 2005, 13: 6105-6110

6 Uzunbajakava N, Lenferink A, Kraan Y, et al. Nonresonant confocal Raman imaging of DNA and protein distribution in Apoptotic cells. Biophys J, 2003, 84: 3968-3981

7 Creely C M, Mercadal S, Volpe G, et al. Raman imaging of neoplastic cells in suspension. Proceedings of the 3rd Optical Trapping and Optical Micromanipulation, 2006 Aug 30, San Diego. SPIE 6326: 63260U

8 Puppels G J, M de Mul F F, Ott O C, et al. Studying sinlge living cells and chromosomes by confocal Raman microspectroscopy. Nature, 1990, 347: 301-303

9 Xie C G, Mace J, Dinno M A, et al. Identification of single bacterial cells in aqueous solution using confocal laser tweezers Raman spectroscopy. Anal Chem, 2005, 77: 4390-4397

10 Yuan Y F, Tao Z H, Liu J X, et al. Raman tweezers-based analysis of carotenoid synthesis in rhodotorula glutinis. Spectrosc Spect Anal, 2011, 31: 1001-1005

11 Snook R D, Harvey T J, Faria E C, et al. Raman tweezers and their application to the study of singly trapped eukaryotic cells. Integr Biol, 2009, 1: 43-52

12 Bankapur A, Zachariah E, Chidangil S, et al. Raman tweezers spectroscopy of live, single red and white blood cells. PLoS One, 2010, 5: e10427

13 Bayden R W, Peter C, Gerwin J P, et al. Resonance Raman spectroscopy of red blood cells using near-infrared laser excitation. Anal Bioanal Chem, 2007, 387: 1691-1703

14 Rao S, Balint F, Cossins B, et al. Raman study of mechanically induced oxygenation state transition of red blood cells using optical tweezers. Biophys J, 2009, 96: 209-216

15 Neugebauer U, Bocklitz T, Clement J H, et al. Towards detection and identification of circulating tumour cells using Raman spectroscopy. Analyst, 2010, 135: 3178-3182

16 Chan J, Taylor D S, Lane S M, et al. Nondestructive identification of individual leukemia cells by laser trapping Raman spectroscopy. Anal Chem, 2008, 80: 2180-2187

17 Tim J H, Elsa C F, Alex H, et al. Spectral discrimination of live prostate and bladder cancer cell lines using Raman optical tweezers. J Biomed Opt, 2008, 13: 064004 
18 Dochow S, Krafft C, Neugebauer U, et al. Tumour cell identification by means of Raman spectroscopy in combination with optical traps and microfluidic environments. Lab Chip, 2011, 11: 1484-1490

19 Banerjee H N, Zhang L. Deciphering the finger prints of brain cancer astrocytoma in comparison to astrocytes by using near infrared Raman spectroscopy. Mol Cell Biochem, 2007, 295: 237-240

20 Chen K, Qin Y, Zheng F, et al. Diagnosis of colorectal cancer using Raman spectroscopy of laser-trapped single living epithelial cells. Opt Lett, 2006, 31: 2015-2017

21 Singh G P, Creely C, Volpe G, et al. Raman spectroscopy of a single living cell in environmentally stressed conditions. Proceedings of the 2nd Optical Trapping and Optical Micromanipulation, 2005 Jul 31, San Diego. SPIE 5930: 1-11

22 Chan J W, Taylor D S, Zwerdling T, et al. Micro-Raman spectroscopy detects individual neoplastic and normal hematopoietic cells. Biophys J, 2006, 90: 648-656

23 Huang W E, Ward A D, Whiteley A S. Raman tweezers sorting of single microbial cells. Environm Microbiol Rep, 2009, 1: 44-49

24 Chan J W, Esposito A P, Talley C E, et al. Reagentless identification of single bacterial spores in aqueous solution by confocal laser tweezers Raman spectroscopy. Anal Chem, 2004, 76: 599-603

25 Mannie M D, Mcconnell T J, Xie C, et al. Activation dependent phases of $\mathrm{T}$ cells distinguished by use of optical tweezers and near infrared Raman spectroscopy. J Immunol Methods, 2005, 297: 5360

26 Abraham S, Marcel J E G. Smoothing and differentiation of data by simplified least squares procedures. Anal Chem, 1964, 36: 16271639

27 Chad A L, Anita M J. Automated method for subtraction of fluorescence from biological Raman spectra. Appl Spec, 2003, 57: 13631367

28 Gao H X. Applied Multivariate Statistical Analysis (in Chinese). Beijing: Peking University Press, 2005

29 John R F, Kazuo N, Chris W B. Introductory Raman Spectroscopy. 2nd ed. Beijing: Academic Press, 2002

30 Xiong Y L, Ye A P, Wen C, et al. Investigation on activating individual living monocytic U937 cell by interleukin-6 using Raman tweezers. Chin Opt Lett, 2010, 8: 1015-1018

31 Bayden R W, Peter C, Gerwin J P, et al. Resonance Raman spectroscopy of red blood cells using near-infrared laser excitation. Anal Bioanal Chem, 2007, 387: 1691-1703

32 Rao S, Balint F, Cossins B, et al. Raman study of mechanically induced oxygenation state transition of red blood cells using optical tweezers. Biophys J, 2009, 96: 209-216

Open Access This article is distributed under the terms of the Creative Commons Attribution License which permits any use, distribution, and reproduction in any medium, provided the original author(s) and source are credited. 